\title{
DIABETIC KETOACIDOSIS IN PREGNANCY: A CASE REPORT
}

Purushotaman Jajuํㅜ J. Aishwarya ${ }^{2}$

\section{HOW TO CITE THIS ARTICLE:}

Purushotaman Jaju, J. Aishwarya. "Diabetic Ketoacidosis in Pregnancy: A Case Report". Journal of Evolution of Medical and Dental Sciences 2014; Vol. 3, Issue 39, August 28; Page: 9964-9967,

DOI: $10.14260 /$ jemds/2014/3292

ABSTRACT: Diabetic ketoacidosis is a serious metabolic complication and its occurrence in pregnancy compromises the life of fetus and mother profoundly. Here by a case of G4P3L3 with term gestation with intrauterine death with diabetic ketoacidosis in labor is reported and the management done for the successful outcome of mother is discussed. Prevention, early recognition, hospitalization and management remain the cornerstones to minimize the outcomes of this dreaded condition.

KEYWORDS: Diabetic ketoacidosis (DKA), intrauterine death, diabetes.

INTRODUCTION: Diabetic ketoacidosis is an infrequent and is a serious metabolic complication of diabetes with high mortality if undetected. Its occurrence in pregnancy compromises both the fetus and the mother profoundly. Although predictably more common in patients with type 1 diabetes, ${ }^{1-3}$ it has been recognized in those with type 2 diabetes as well as gestational diabetes. It usually occurs in later stages of pregnancy.

We report a case of diabetic ketoacidosis who is a known case of type 2 diabetic mellitus and Presented with intrauterine fetal demise.

CASE REPORT: A 33 yr. old G4P3L3 with history of diabetes mellitus since 4 yrs., diagnosed after her third delivery. She was referred to our hospital at her 38th week of gestation in view of uncontrolled diabetes with intrauterine fetal demise. She came with complaints of vomiting episodes and giddiness on and off since 2 days. She gave a history of not perceiving fetal movements since $12 \mathrm{hrs}$. prior to admission and back pain since $10 \mathrm{hrs}$. On direct questioning she also has polydipsia and polyuria. She was dehydrated, had tachycardia, tachypnea and afebrile.

Her menstrual cycles were regular and was 38 weeks 3 days pregnant. She was married for 10 yrs., was diagnosed with GDM during her third pregnancy which was persistent in her postpartum period and was diagnosed with type 2 DM. She was put on inj insulin actrapid 6-6-8 units. Her antenatal visits were irregular during the present pregnancy and her sugar levels were not controlled well. She had 2 ultrasonography scans done during her $2^{\text {nd }}$ and 3 rd trimester of pregnancy which showed minimal hydramnios and no anomalies were detected.

On examination, Patient was conscious and oriented, well-built and nourished, dehydration was present. No pallor, mild pedal oedema+.PR-98/min, tachycardia+, BP-100/70 mm Hg RR-24/min CVS-S1S2+, RS-B/L air entry+, mild tachypnea, P/A-uterus term size, acting 2C/25"/10', cephalic presentation and FHS-absent, P/V-Cx-partially effaced, os-3-4cm, Vx--1station, membranes were present and pelvis was adequate.

Her investigations revealed CBC-TC-14, 000, DC-Neutrophils-60\%, lymphocytes-35\%, basophils and eosinophils-nil.HB-10 g/dl, Platelets-2 lakhs.

Urine analysis-sugars4+, ketone bodies3+, proteins+, pus cells-15-20/hpf and RBS-305mg/dl, HBA1C-8. 
Arterial blood gas analysis showed severe metabolic acidosis, PH-7.16 (7.35-7.45), carbon dioxide tension of 1.5(4.7-6.0) kPa, oxygen tension 14.6 (>10.6) kPa Bicarbonate-3.5 (22-28)mm0l/L, Lactate-2.9 (0.6-1.7) mmol/L, Serum Na-126(135-145) mmol/L, Serum K-5.5 (3.5-5.0) mmol/L, Urea$25 \mathrm{mg} / \mathrm{dl}$ and creatinine- $1.1 \mathrm{md} / \mathrm{dl}$.

Patient was immediately resuscitated with intravenous fluids (normal saline) and oxygen. She was started on insulin, initially with 10 units subcutaneously and 20 units in infusion pump and maintained according to sliding scale for DKA. Her sugar levels, electrolytes and arterial blood gas analysis were serially monitored. Intravenous antibiotics were given in view of possible urinary tract infection. Once the metabolic acidosis was corrected she was treated with subcutaneous insulin on regular regimen.

Artificial rupture of membranes was done once cervix was $6 \mathrm{~cm}$ dilated and a fresh stillborn female baby weighing $3.9 \mathrm{~kg}$ was delivered. Placenta and membranes were delivered completely. No PPH. Her postpartum sugars were kept under control with an insulin regimen of 8-6-6units and was followed up for 6 weeks.

DISCUSSION: DKA affects only $1 \%$ of diabetic pregnancies with fetal loss rate of $9 \% .{ }^{4}$ It usually occurs in later half of pregnancy because of insulin antagonistic state ${ }^{5}$, accelerated starvation and lowered buffering capacity and usually compromises the fetus. Despite improvement in its incidence rates and outcomes over the years, it still remains as a major clinical problem as it occurs more rapidly in non-pregnant patients often causing delay in the diagnosis.

The incidence rate of diabetic ketoacidosis in pregnancy and the corresponding fetal mortality rates from different retrospective studies are summarized in the table 1.6-9 During pregnancy various factors can precipitate ${ }^{10-11}$ and contribute to the occurrence of DKA. In this case DKA might have been triggered by vomiting, secondary to UTI. A high glycosylated hemoglobin shows that the patient had uncontrolled sugar levels during her antenatal period.

\begin{tabular}{|c|c|c|c|}
\hline REFERENCE & PERIOD & INCIDENCE & FETAL MORTALITY \\
\hline Lufkin et al & $1950-79$ & $18 / 277(7.9 \%)$ & $5 / 18(27.7 \%)$ \\
\hline Kilvert et al & $1971-90$ & $11 / 635(1.73 \%)$ & $22 \%$ \\
\hline Chauhan et al & $1976-81$ & $51 / 227(22 \%)$ & $35 \%$ \\
\hline Cullen et al & $1985-95$ & $11 / 520(2 \%)$ & $1 / 11(9 \%)$ \\
\hline & $\begin{array}{c}\text { Table 1: Incidence and fetal mortality rates in diabetic } \\
\text { pregnancies complicated by diabetic ketoacidosis }\end{array}$ \\
\hline
\end{tabular}

There are various mechanisms for fetal demise such as decrease in uteroplacental blood flow due to: (a) osmotic diuresis leading to volume depletion and (b) maternal acidosis that can cause fetal hypoxic insult. Maternal acidosis could lead to fetal acidosis and electrolyte imbalance. Maternal hypokalemia and fetal hyperinsulinaemia if severe could cause fetal hypokalemia leading to fetal myocardial suppression and fatal arrhythmia.

Maternal hypophosphatemia associated with diabetic ketoacidosis can cause decrease in 2, 3diphosphoglycerate leading to impaired delivery of oxygen to the fetus. Fetal hyperinsulinaemia resulting from maternal hyperglycemia increases fetal oxygen requirement by stimulating oxidative metabolic pathway. ${ }^{12-15}$ 
CONCLUSION: While the outcomes of diabetic ketoacidosis in pregnancy have improved over the years, significant maternal and fetal mortality still remains. Prevention, early recognition, hospitalization and aggressive management remain the cornerstones to minimize the outcomes of this dreaded complication.

\section{REFERENCES:}

1. Bedalov A, Balasubramanyam A. Glucocorticoid-induced ketoacidosis in gestational diabetes: sequelae of acute treatment of preterm labor. Diabetes Care 1997; 20: 922-4.

2. Maislos M, Harman-Bohem I, Weitzman S. Diabetic ketoacidosis. A rare complication of gestational diabetes. Diabetes Care 1992; 16: 661-2.

3. Bernstein IM, Catalano PM. Ketoacidosis in pregnancy associated with the parenteral administration of terbutaline and betamethasone: a case report. J Reprod Med 1990; 35: 818.

4. American Diabetes Association. Report on the expert committee on the diagnosis and classification of diabetes mellitus. Diabetes Care 1999; 22(suppl 1): S5.

5. Catalano PM, Tyzbir ED, Roman NM, et al. Longitudinal changes in insulin release and insulin resistance in nonobese pregnant women. Am J Obstet Gynecol 1991; 165: 1667-72.

6. Lufkin EG, Nelson RL, Hill HM, et al. Analysis of diabetic pregnancies at Mayo Clinic 1950-1979. Diabetes Care 1984; 7: 539-47.

7. Kilvert JA, Nicholson Ho, Wright AD. Ketoacidosis in diabetic pregnancy. Diabet Med 1993; 10: 278-81.

8. Chauhan SP, Perry KG Jr, McLaughlin BN, et al. Diabetic ketoacidosis complicating pregnancy. J Perinatol 1996; 16: 173-5.

9. Cullen MT, Reece EA, Homko CJ, et al. The changing presentations of diabetic ketoacidosis during pregnancy. Am J Perinatol 1996; 13: 449-51.

10. Montoro MN, Myers VP, Mestman JH, et al. Outcome of pregnancy in diabetic ketoacidosis. Am J Perinatol 1993; 10: 17-20.

11. Rodgers BD, Rodgers DE. Clinical variables associated with diabetic ketoacidosis in pregnancy. J Reprod Med 1991; 32: 797-800.

12. Belcher JN, Stringer VG, Prystowsky M. Blood flow to the human uterus during maternal metabolic acidosis. Am J Obstet Gynecol1975; 121: 789-94.

13. Kitzmiller JL. Diabetic ketoacidosis and pregnancy. Contemp Obstet Gynecol 1982;20:141-7.

14. Ditzel J, Standl E. The oxygen transport system of red blood cells during diabetic ketoacidosis and recovery. Diabetologia 1975; 11: 255-60.

15. Philipps AF, Rosenkrantz TS, Raye J. Consequences of perturbations of fetal fuels in ovine pregnancy. Diabetes 1985; 54: 32-5. 


\section{AUTHORS:}

1. Purushotaman Jaju

2. J. Aishwarya

\section{PARTICULARS OF CONTRIBUTORS:}

1. Professor and HOD, Department of Obstetrics and Gynaecology, BLDE University's Shri B.M. Patil Medical College.

2. Post Graduate, Department of Obstetrics and Gynaecology, BLDE University's Shri B.M. Patil Medical College.

\section{NAME ADDRESS EMAIL ID OF THE CORRESPONDING AUTHOR:}

Dr. J. Aishwarya,

Post Graduate,

Department of Obstetrics and Gynaecology, BLDE University's,

Shri B.M. Patil Medical College,

Bijapur, Karnataka.

Email: aishuj13@yahoo.com

Date of Submission: 08/08/2014.

Date of Peer Review: 09/08/2014.

Date of Acceptance: 21/08/2014.

Date of Publishing: 28/08/2014. 